\title{
Research on Visible Light Communication Technology
}

\author{
Shiyan Yang ${ }^{1, a}$ \\ ${ }^{1}$ College of Electronics and Information Engineering , Sichuan University, Sichuan 610065, China \\ ayangshiyan0605@163.com
}

Keywords: visible light communication, white LED, light-emitting diode, channel coding, smart light.

\begin{abstract}
Visible light communication system uses white light emitting diode (LED) as light source, so the system has the dual role of communication and lighting and has high transmit power without taking radio spectrum, electromagnetic interference and electromagnetic radiation, what's more, it could save energy efficiently and so on. This paper describes the characteristics of visible light communication technology, introduces the visible light communication framework and discusses some methods which can improve the performance and quality of visible light communication. Finally, the future application of visible light communication is prospected.
\end{abstract}

\section{Introduction}

Visible Light Communication (VLC) is a way of communication that uses visible light as information carrier to transmit optical signal in the air without wired channel like optical fiber. It utilizes high speed flashing signal to transmit information, and the system can cover as far as the light reaches with a high speed Internet wire device attached to the lighting device, which means we can have high speed Internet access as long as there is light. In addition, Light Emitting Diode (LED) has been widely used in the field of monitor, lighting decoration, lightning and so forth since it was invented in the 1960s, and it is closely related to almost every social member, which means it has huge demand. Moreover, LED has many advantages concerning low power consumption, energy saving and being environmentally friendly. Also it has the features of fast switch, high sensitivity and excellent modulation performance. This is why LED can be used to transmit optical signal in VCL [1].

With the help of all-optical network technology and the skyrocketing demand for broadband mobile communication, indoor VCL has drawn great attention of the academia and industry, and the work of visible light global standardization has been launched. Therefore, VCL is a research program that possesses enormous research value, broad application prospects and great market potential [2].

\section{The feature of VCL}

\subsection{The advantages of VCL}

(1)High speed: the modulation rate of LED is incredibly high so that the human eye cannot sense it flashing at all, which means it can be used both in communication and lightning. (2) Wide spectrum: the width of visible spectrum that VCL uses is quite large, so single data channel can have high bandwidth or it can contain more channels for parallel transmission. In that case the speed of data transmission can increase significantly to the peak of several hundred $\mathrm{MB} / \mathrm{s}$ [3]; (3) High security: light cannot travel through the wall, which means the information indoor is safe for it will not be leaked outside the room. (4) Low cost: currently the cost of LED is quite low and the related technology is mature, which can largely reduce the cost of the application of VCL. In a word, the importance of VCL technology is obvious since the demand for high speed and economical Internet is surprisingly huge. 


\subsection{The unsolved problems of VCL}

(1) Although white LED has high luminous efficiency,at present limited by related technology,the white LED of VCL does not have high enough modulation rate and the modulation bandwidth is only several MHz, which is challenging for future application [4]; (2) Once the light source is blocked or gone, the Internet signal will be cut off immediately; (3) The data is hard to return because the uplink channel and downstream channel are separate; (4) Nowadays the special detector has not been invented, so it is not easy to monitor the operation of the system; (5) There are lots of different optical paths because there are a multitude of light sources located at different places, and the different distances can lead to so-called multipath effect, which will cause severe inter-symbol interference [2]; (6) Additionally, the special integrated chip has not been invented, so the system structure is complex and inefficient.

Overall, VCL is no match for the traditional wireless communication technology so it can only serve as a supplement, and these two means can develop together by complementing one another. However, if the above-mentioned problems can be solved properly, then VCL will also have infinite developing prospect.

\section{The framework of VCL system}

One of the typical design of indoor VCL system is shown in Figure 1. It consists of terminal, VCL adapter, white LED light source, VCL hubs, photoelectric receiver and corresponding signal processing unit. The system is composed of two parts: forward link and reverse link, and each of them contains emission and reception part. The emission part includes white LED light source and corresponding signal processing unit, while the reception part is made of photoelectric detector and corresponding signal processing unit [5].

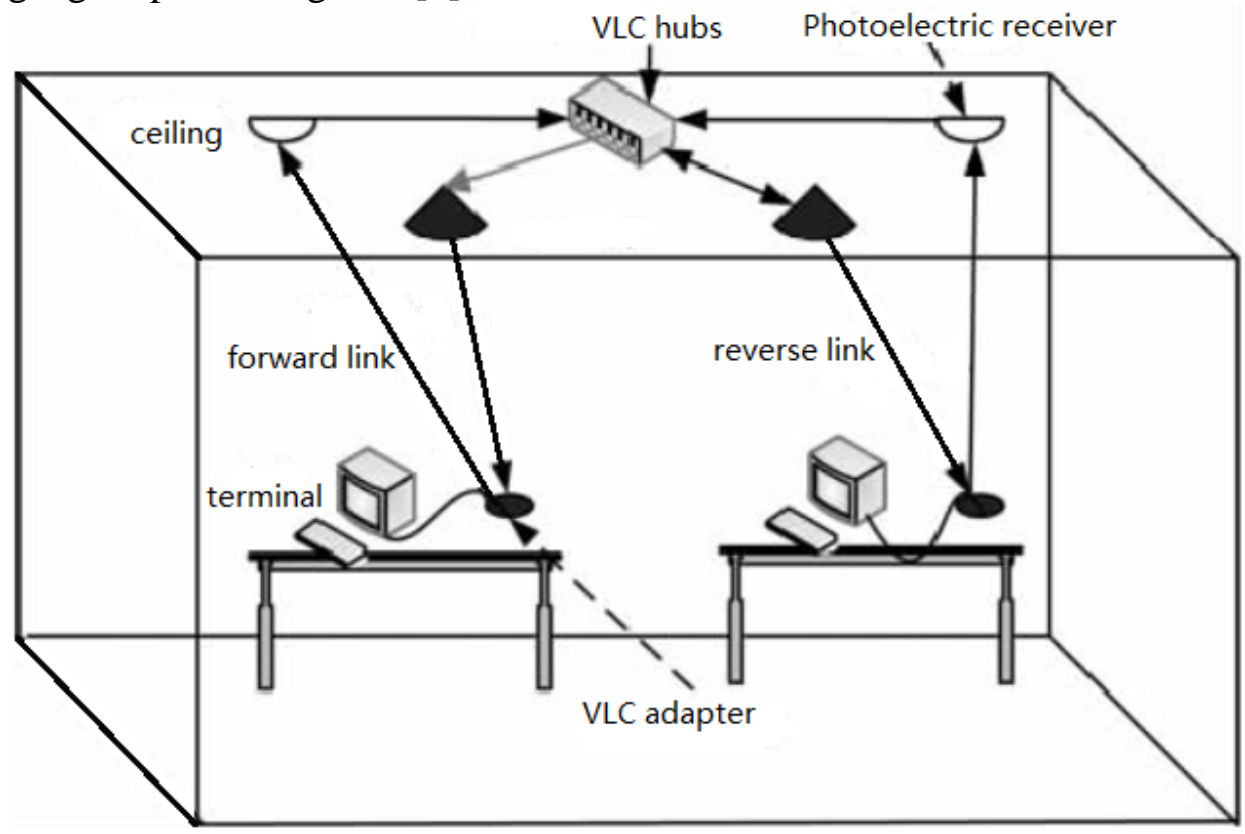

Fig.1 The illustration of indoor VCL system

VCL hubs receive the information from the end-user, and meanwhile broadcast the information received through the main light source periodically. VCL adapter contains white LED light source of the forward link and photoelectric receiver of the reverse link, and its job is to modulate end-user's information into optical signal and receive the optical signal from reverse link. The photoelectric detector in the ceiling receives the optical signal from the user and convert it into electric signal, then sends it to VCL hubs. After a simple procession in the VCL hubs, the electric signal will become optical signal and be broadcast by white LED light source. At the receive end, the visible light adapter of terminal demodulates the information received and sends it to the end-user. In this way, wireless communication in local area network can be accomplished [5]. 


\section{Modulation and demodulation technology}

\subsection{On-Off Keying}

On-Off Keying (OOK) is a way of modulation that optical transmitter transmits light pulse $p(\mathrm{t})$ within the period of $T=1 / R_{b}$ when the bit rate of the source is $R_{\mathrm{b}}$, and uses $p(\mathrm{t})$ to indicate " 1 ", no light pulse to indicate " 0 ". When duty cycle is 1 , the bandwidth is approximately $B_{\mathrm{OOK}}=R_{\mathrm{b}}$. It is easy to accomplish, and the bandwidth efficiency is quite high. Nonetheless, its power efficiency is low.

\subsection{Pulse Position Modulation (PPM)}

Pulse Position Modulation (PPM) is another common way of binary modulation. A source of $\mathrm{k}$ bit $\mathrm{U}=\left(\mu_{1}, \mu_{2}, \ldots \mu_{\mathrm{k}}\right) \in\{0,1\}^{k}$ can get signal $X(0, \ldots 0,1,0, \ldots 0) \in\{0,1\}^{k}$ after M-system PPM. PPM has high power efficiency and low bandwidth efficiency, we can say that it sacrifices bandwidth efficiency for power efficiency.

\subsection{Sub-Carrier Pulse Position Modulation}

The principle of Sub-Carrier Pulse Position Modulation (SC-PPM) is shown in Figure 2. SC-PPM is a recommended way modulation in Japanese Visible Light Communication Standard, and it uses subcarrier to transmit information on the basis of PPM.

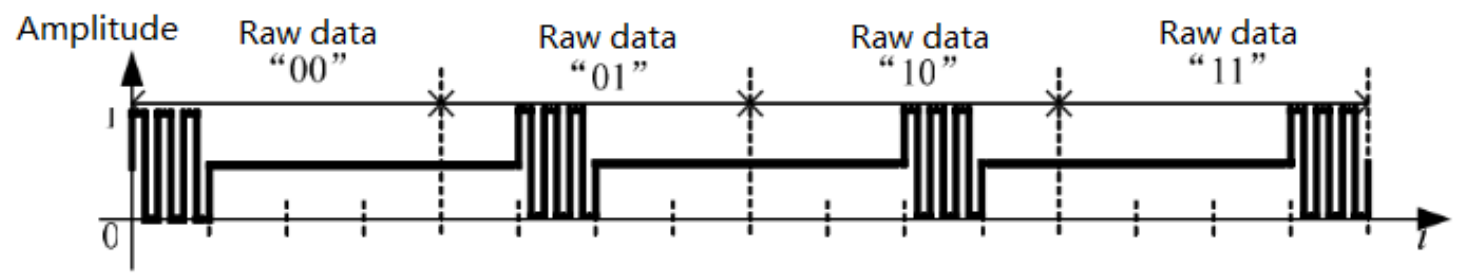

Fig.2 Illustration of Sub-Carrier Pulse Position Modulation

\subsection{Differential pulse position modulation}

Differential pulse position modulation (DPPM) is an improved version of PPM. As long as we remove the time slots that follow the high levels of PPM signs we can get corresponding DPPM signal. The bandwidth requirements, power utilization and missed slot rate are between those of OOK and PPM [8].

\subsection{Color shift keying}

The principle of Color shift keying (CSK) is to utilize the different proportion of color light intensity in each sign to transmit information on condition that the total blending color coordinates remain unchanging. The data after CSK modulation will be converted into visible light signal to transmit information by red, green and blue LED. According to color mixing theory, suppose that the three-color LED can create $n$ kinds of colors, and the information bits of each color is $\log _{2} n$. Meanwhile, to assure that the color of indoor light meets the requirements, the color coordinates of mixed colors need to be controlled. The main advantage of CSK is that its data transfer rate can be higher than 1bit/clock, which makes it high-speed modulation.

\subsection{Orthogonal frequency division multiplexing}

Orthogonal frequency division multiplexing (OFDM) is a kind of multi-carrier data communication technology. It divides high-speed data stream into parallel low-speed data stream, and uses low rate multi-status symbols to modulate mutually orthogonal subcarriers, eventually forms a transmission system that transmits multiple low rate symbols in parallel.

The advantages of OFDM include: (1) It reduces frequency selective fading and narrow band interference; (2) It fights against interference between signal waveforms, so it is suitable for high speed data transmission in multipath environment and fading channel; (3) Owing to the joint coding of each subcarrier, it has strong anti-fading ability; (4) We can use the way based on IFFT/FFT to make it happen.

However, presently OFDM technology still has two flaws: (1) Its peak-to-average power ratio is huge, and it is sensitive to non-linearity in the system; (2) It is sensitive to timing and frequency offset. 


\section{Application of Visible Light Communication Technology}

\subsection{Indoor visible light communication}

(1) Indoor wireless network. We can have access to the high speed Internet through all kinds of terminal equipment (TV, tablet, mobile phone, etc.) and get the instant information as soon as we turn on the light. Moreover, the family members can share data and information with each other even faster.

(2) Indoor Positioning. Traditional satellite positioning method is hard to accomplish the precise positioning of indoor mobile users, while VCL can transmit the information concerning user's location via LED, which can accomplish the precise indoor positioning. Currently, many indoor positioning programs based on VCL have been put forward, and Visible Light Communication Consortium (VLCC) in Japan has achieved the goal of indoor positioning experiment based on supermarket environment [3].

\subsection{Outdoor visible light communication}

(1) Intelligent Traffic System (IST).Nowadays cars mostly use LED for lightning, which can help build communication links among cars, traffic control center and traffic light. By doing this we can improve traffic efficiency, ease traffic congestion, avoid traffic accidents and reduce energy consumption and environmental pollution.

(2) Underwater communication. VCL technology can overcome the problems of underwater acoustic communication technology such as narrow bandwidth, environmental impact, low applicable carrier frequency and large transmission delay, which will enable the underwater work to be safer and more efficient. In 1979,US firstly put forward the ideal of utilizing blue and green laser of $0.498 \mu \mathrm{m}$ to communicate with submarine. At the end of 1983, Soviet Union accomplished the experiment of emitting blue laser beam to space track reflector and then reflecting it to underwater ballistic submarine. In 2008, F. Hanson and others in US accomplished underwater optical communication experiment that had a transmission rate of $1 \mathrm{~Gb} / \mathrm{s}$ for the first time in the lab. In February 2010, The Woods Hole Oceanographic Institution in US reached underwater optical communication rate of $10 \sim 20 \mathrm{Mb} / \mathrm{s}$ within $100 \mathrm{~m}$. However, so far commercial underwater optical communication technology is not mature.

\section{Conclusion}

Visible Light Communication can both be used for lightning and communication, and it has the advantages of high transmission speed, high security, no electromagnetic interference and so forth. It is an ideal indoor high-speed wireless access program. VCL has become a global research hotspot, even though there are some problems remained to be solved, a growing number of solutions are being put forward. For instance, the fractionally spaced equalization (FSE) technology is investigated to mitigate the effects of inter-symbol interference, and dual duration differential pulse position modulation (DD-DPPM) is proposed on the basis of high bandwidth, high power and low slot error rate requirements of VLC system in vehicle. Therefore, in the future VCL will become a vital part of the communication field and great boost to the information society.

\section{Reference}

[1] S Guo, L Zang, M Han, H Cheng, F Zhong. Interval pulse position modulation in visible light communication [J]. Optics and Precision Engineering, 2014, 22 (7): 1760-1765.

[2] J Wang, X Xie, L Cao, M Sheng, M Feng. Fractionally spaced equalization for indoor visible light communication system [J]. Optics and Precision Engineering, 2012, 20 (1): 24-30.

[3] T Chen, L Liu, W Hu. Visible light communication [J]. ZTE Technology Journal, 2013, 19 (1): 49-52.

[4] X Che, Z Liang, X Liu. Receiving characteristics of indoor MIMO visible light communication [J]. Chinese Journal of Luminescence, 2016, 37 (2): 242-249. 
[5] H Luo, C Chen, Q Fu, H Zhao, C Mo. Key technologies of indoor visible light communication based on white LED [J]. Optical Communication Technology, 2011, 35 (2): 204-204.

[6] W Yun. Study on modulation and demodulation method for visible-light communication system [D]. Changchun University of Science and Technology, 2008.

[7] J Liu. PPM modulation and coding techniques of visible light communications system [D]. Southeast University, 2016.

[8] X Song, S Jia, Z Zhao, Y Wei. Dual duration differential pulse position modulation based on visible light communication [J]. Journal of Armored Force Engineering Institute, 2016, 30 (3): 79-82.

[9] Q Zhu, Y Tang, L Cui, J Luo, X Zhang, H Huang. A CSK-HVPM integrated modulation method for visible light communication [J]. Optical Technique, 2016, 42 (6): 561-566.

[10] Y Wang, M Zhou, Z Song. Development of underwater wireless communication Technology [J]. Communications Technology, 2014, 6: 589-594. 